\section{Opioid-Hoch im Norden}

Offenbar werden zu häufig und zu unkritisch Fentanylpflaster eingesetzt $(40,8 \%$ aller Tagesdosen), so eine Studie zur Versorgung mit starken Opioiden der WHO-Stufe III für das Jahr 2011. Die transdermale Anwendung ist ausschließlich bei stabilem Schmerzmittelbedarf und opioidtoleranten Patienten indiziert. Sie erfolgt in der Praxis allerdings in mehr als der Hälfte der Fälle (52,9\%), ohne dass vorher ein anderes Opioid eingesetzt wurde. Der überwiegende Teil der Opioid- und auch Fentanylverschreibungen erfolgte 2011 durch Hausärzte. Insgesamt wurden 18,9 Mio. definierte
Tagesdosen (DDD) starker Opioide verordnet, durchschnittlich 208,6 DDD pro 100 Versicherten und einem auffälligen Nord-Süd-Gefälle (s. Abb.) mit Werten zwischen 259,5 in Niedersachsen und 145,9 DDD in Baden-Württemberg. Bei mehr als der Hälfte der Neuverordnungen wurden höhere Dosierungen als die niedrigste verfügbare $\mathrm{zu} 12,5 \mu \mathrm{g} / \mathrm{h}$ eingesetzt. Verwendet wurden Routinedaten der 9,1 Mio. Versicherten der BARMER GEK.

Hoffmann Fet al, Verordnung von starken Opioiden im Jahr 2011. Schmerz 2012, 26:707-714

\section{Frau braucht weniger Analgesie}

\title{
Lässt chronischer Schmerz das Hirn schwinden?
}

Bei chronischen Schmerzen verändert sich auch das Gehirn: Manche Hirnarale werden dünner, sodass man hier bleibende Schäden befürchtet. Allerdings sind diese Veränderungen reversibel: Nach einer erfolgreichen Schmerztherapie normalisiert sich das Volumen. Die Verminderung der grauen Substanz lässt sich bei fast allen $\mathrm{Pa}$ tienten mit chronischen Schmerzen beobachten, egal ob es sich um Kopf-, Rückenoder Phantomschmerzen handelt, berichtete Prof. Dr. Arne May, Schmerzexperte vom Uniklinikum Hamburg-Eppendorf, beim Fortbildungsveranstaltung „Neuro Update“ in Mainz. Je länger die Patienten erkrankt sind, umso ausgeprägter sei die Verminderung der grauen Substanz. Lange Zeit habe man befürchtet, dass es sich dabei um eine irreversible Atrophie oder einen dauerhaften Schaden handelt, der möglicherweise auch kognitive Funktionen beeinträchtigt und eine Demenz begünstigt. Hier könne man nun Entwarnung geben. Die hirnstrukturellen Veränderungen sind bei einer erfolgreichen Schmerztherapie offenbar vollkommen reversibel. (mut)

Neuro Update, Mainz 2013, Vortrag "Schmerz/Kopfschmerz" von Prof. Dr. Arne May, Uniklinikum Hamburg-Eppendorf

\section{Mit Schmerzmittel zum Nierenkrebs}

Immer mehr Studien attestieren insbesondere ASS, aber auch (anderen) NSAR eine krebsvorbeugende Wirkung, unter anderem gegenüber Karzinomen des Kolorektums, der Brust und der Prostata. Ein Schutz vor Nierenkrebs lässt sich jedoch nicht feststellen. Die meisten Analgetika scheinen das Erkrankungsrisiko sogar zu fördern, wie eine Metaanalyse jetzt ergeben hat. Die Analyse fußt auf Daten von 20 Studien aus Nordamerika, Europa und Australien, davon zwölf Fall-Kontroll-Studien mit 7075 Erkrankten und 579.285 Kontrollen und acht Kohortenstudien mit 1165 Erkrankten unter 579.285 Teilneh- mern. Danach ist die gelegentliche oder regelmäßige Anwendung von Paracetamol, die in 14 Studien ausgewertet wurde, mit einem Anstieg des Nierenkrebsrisikos um $28 \%$ verbunden. Höhere Dosierungen gingen mit einem höheren Risiko einher (plus $68 \%$ ). Anwender von NSAR ohne ASS, sie wurden in fünf Studien untersucht, hatten insgesamt eine um 25\% erhöhte Nierenkrebsrate. Auch hier zeigte sich eine DosisWirkungs-Beziehung mit einer Steigerung der Krebsrate um 56\% bei Einnahme hoher Dosen.

(bs)

Choueiri TK et al, Int J Cancer 2013 (online first)

\section{Beethoven gegen Intensiv-Schmerz}

Zwei Stunden Klassik auf die Ohren reduziert den Stress auf der Intensivstation: Beatmete Patienten brauchen weniger Sedativa und Schmerzmittel, so das Ergebnis einer kanadischen placebokontrollierten Studie mit 55 Patienten. Neben den Vitalzeichen wurde der Bedarf an Sedativa und Schmerzmitteln, Entzündungsparameter sowie Prolaktin, Kortisol und ACTH festgehalten. Während es bei den Vitalzeichen keine nennenswerten Unterschiede zwischen den Gruppen mit und ohne Musik gab, deutete sich beim Fentanylbedarf ein Trend zur Signifikanz an. Dieser ging nach dem $\mathrm{Mu}$ sikhören zurück (von 1600 auf $1350 \mu \mathrm{g}$ ). Gab es dagegen nur Kopfhörer ohne Musik auf die Ohren, stieg der Bedarf (von 1600 auf $1750 \mu \mathrm{g}$ ). Ähnliches war auch beim Sedativa- und Hypnotikakonsum zu beobachten, allerdings waren hierbei die Unterschiede noch geringer. Kortisol ging von $815 \mathrm{nmol} / \mathrm{l}$ vor dem Musikhören auf $741 \mathrm{nmol} / \mathrm{l}$ danach zurück, ohne Musik blieben die Werte konstant, allerdings waren die Unterschiede auch hier nicht signifikant. Bei zwei Drittel der Patienten sackten die Kortisolwerte nach dem Musikhören aber deutlich ab (<600 nmol/l).

(mut)

Beaulieu-Boire G et al, J Crit Care 2013 (online first) 